\title{
LA INFLUENCIA DEL GÉNERO EN LA DIRECCIÓN DE LAS SOCIEDADES COOPERATIVAS ESPAÑOLAS SOBRE LA RENTABILIDAD Y EL ENDEUDAMIENTO: UN ANÁLISIS EMPÍRICO ${ }^{1}$
}

\author{
POR \\ Carmen María HERNÁNDEZ NICOLÁS, \\ Juan Francisco MARTÍN UGEDO y \\ Antonio MINGUEZ VERA ${ }^{2}$
}

\section{RESUMEN}

En el presente trabajo se aborda la reclamación por el mercado de una nueva gestión empresarial que asegure la presencia de la mujer en la toma de decisiones para dar respuesta a las nuevas necesidades sociales. Así, este trabajo analiza la influencia de la diversidad de género de los consejeros sobre la rentabilidad y el nivel de endeudamiento para una muestra de 5.199 cooperativas españolas. A diferencia de las sociedades capitalistas, estas organizaciones presentan una serie de peculiaridades en su gobierno, ya que los socios son a su vez principales, agentes y clientes. El estudio se centra en el contexto español, donde existe un debate abierto sobre la importancia de la gestión empresarial femenina impulsado, como en otros países, por la proliferación de legislación sobre la igualdad de género, siendo, además, España el país pionero en contar con una legislación específica sobre Economía Social. Los resultados muestran que las cooperativas con mayor representación femenina en su Consejo Rector presentan una mayor rentabilidad. Por otro lado, en aquellos consejos con un mayor porcentaje de mujeres muestran un menor nivel de endeudamiento.

Palabras clave: Consejo Rector, Mujer, Economía Social, Variables Financieras.

Claves ECONLIT: J54, J16.

\footnotetext{
${ }^{1}$ Este trabajo es parte del Proyecto FEM2013-40578-P financiado por el Ministerio de Economía y Competitividad. También agradecemos el apoyo de la Fundación CajaMurcia.

${ }^{2}$ Universidad de Murcia, España. Departamento de Organización de Empresas y Finanzas. Direcciones de correo electrónico: cm.hernandeznicolas@um.es, juanfran@um.es y minver@um.es.

REVESCO No 122 - Tercer Cuatrimestre 2016 - ISSN: 1885-8031 - www.ucm.es/info/revesco

http://dx.doi.org/10.5209/rev_REVE.2016.v122.52021

Fecha de recepción: 05/06/2015

Fecha de aceptación: 16/02/2016
} 


\title{
THE INFLUENCE OF GENDER IN THE DIRECTION OF SPANISH COOPERATIVE SOCIETIES ON PROFITABILITY AND INDEBTEDNESS: AN EMPIRICAL ANALYSIS
}

\begin{abstract}
In this paper the claim for the market for a new business management to ensure the presence of women in decision -making to respond to new social needs addressed. Thus, this paper analyzes the influence of gender diversity of the directors on the profitability and the level of debt for a sample of 5,199 Spanish cooperatives. Unlike capitalist societies, these organizations have a number of peculiarities in their government, and that the partners are themselves major time, agents and customers. The study focuses on the Spanish context, where there is an open debate on the importance of women's business management, as in other countries, driven by the proliferation of legislation on gender equality, being, in addition, Spain, the pioneer in having specific legislation on Social Economy. The results show that cooperatives with greater female representation in theirs Boards have higher profitability. On the other hand, those Boards with a higher percentage of women show a lower level of indebtedness.
\end{abstract}

Keywords: Gender, cooperatives, returns, debt.

\section{INTRODUCCIÓN}

El mercado está reclamando un modelo de gestión empresarial que considere el impacto de sus actuaciones no sólo en su dimensión económica, sino también en la social y medioambiental. Una serie de iniciativas empresariales, cuyo principal exponente es el cooperativismo, nacen con el objetivo de resolver las nuevas necesidades sociales que han aparecido en los últimos decenios, y han originado la revitalización de la Economía Social. (Álvarez, López y Cantó, 2000).

España ha sido el país pionero en contar con una legislación específica sobre Economía Social (Ley 5/2011, de 29 de marzo. Publicada en BOE 76 de 30 de marzo de 2011). En ella, se define a la Economía Social como el conjunto de actividades económicas y empresariales que, en el ámbito privado, llevan a cabo entidades que persiguen el interés general económico o social, o ambos. Estas entidades se rigen por unos principios orientadores entre los que cabe destacar la primacía de las personas y del fin social sobre el 
capital, la promoción de la solidaridad interna y con la sociedad que favorezca la igualdad de oportunidades, la cohesión social.

Las cooperativas son sus representantes más genuinos. La actual Ley de Cooperativas (Ley 27/1999, de 16 de julio. Publicada en BOE 170 de 17 de Julio de 1999) define a las cooperativas como sociedades constituidas "por personas que se asocian, en régimen de libre adhesión y baja voluntaria..., con estructura y funcionamiento democrático, conforme a los principios formulados por la Alianza Cooperativa Internacional (International Cooperative Alliance, 2012)". Todos los socios están unidos a la cooperativa por un contrato de sociedad (Alonso, 1984), por lo que son empresarios. La puesta en práctica de sus valores (autoayuda, autorresponsabilidad, democracia, igualdad, equidad y solidaridad) se rige por los principios cooperativos. De estos principios afectan principalmente al gobierno corporativo los siguientes: El principio de adhesión voluntaria y no discriminación, por el que no hay discriminación social, política, religiosa, racial o de género. El principio de gestión democrática, por el que los socios participan en la fijación de políticas y la toma de decisiones, "un socio un voto" (Herrera y Sanchís, 1993) y el principio de educación, formación e información. El problema de gestión que se presenta en las cooperativas por la compleja relación de estas organizaciones con sus miembros (Nilsson, 1996) puede estudiarse a través de la teoría de la agencia (Jensen y Meckling, 1976) y este es el enfoque que se emplea en este trabajo.

En España, en los últimos años, el número de cooperativas ha crecido a un ritmo rápido. Por sus principios rectores, algunos autores afirman que estas entidades son un instrumento eficaz dónde las mujeres pueden ascender dentro de la organización jerárquica (Mateos, Iturroiz y Gimeno, 2009), con implicación directa en la gestión (Mateos, Escot y Gimeno, 2006; 2010; Mateos et al., 2009), pudiendo conseguir sus metas (García, 2010).

Una de las áreas que han sido ampliamente estudiadas en el ámbito empresarial es la presencia de mujeres en los Consejos de Administración. Terjesen, Sealy y Singh (2009) hicieron un estudio exhaustivo con una revisión de más de 400 publicaciones, 180 de ellos son artículos y libros académicos. Grosvold y Brammer (2011) clasificaron todas estos estudios en tres categorías: micro (directores individuales), meso (empresas u organizaciones) y macro (la industria dentro de la cual se encuentran las organizaciones). La mayoría de estos estudios está en los niveles micro y meso. 
No sólo en el terreno académico la presencia de la mujer en el Consejo se ha convertido en un tema de actualidad, sino también en el terreno social. Por lo tanto, en los últimos años se ha producido una presión de la sociedad a fin de incluir a las mujeres en los Consejos de Administración. En consecuencia, el promedio del número de consejeras ha aumentado en Europa. Sin embargo, la representación femenina está todavía en un nivel bajo, existiendo una amplia variación entre países, siendo lo países nórdicos los que tienen, en promedio, una mayor representación de mujeres en los consejos (Heidrick y Struggles, 2011; Mateos de Cabo, Gimeno y Escot, 2011).

El Consejo de Administración tiene cuatro funciones importantes: seguimiento y control de los gestores, proporcionar información y asesoría a los consejeros, supervisar el cumplimiento de leyes y reglamentos aplicables y vincular las corporaciones con el entorno (Mallin, 2004). La efectividad del Consejo de Administración depende de varios factores, entre ellos, la cualificación y experiencia de los miembros del Consejo, su posible implicación en múltiples cargos, su nivel de propiedad de acciones y el tipo de esquema de remuneraciones empleado (Campbell y Mínguez-Vera, 2008).

La evidencia sostiene que las consejeras pueden tener una influencia importante en el estilo de trabajo y los procesos del Consejo y, consecuentemente, en el rendimiento de la compañía (Kesner, 1988; Bilimoria y Piderit, 1994; Farrell y Hersch, 2005). De hecho, Huse, Nielsen y Hagen (2009) apuntan que el principal argumento para la promoción de las mujeres es que la diversidad es importante para la creación de valor en la empresa. Sin embargo, la evidencia del análisis de la relación entre la diversidad de género en el Consejo y el rendimiento de la empresa es reducida.

Carter, D’Souza, Simkins y Simpson (2010) en una revisión de la teoría y la evidencia empírica previa, localizaron solamente siete estudios que, específicamente, analizaban la relación entre la diversidad de género en el Consejo y el rendimiento de la empresa (Shrader. Blackburn y Iles, 1997; Carter, Simkin y Simpson, 2003; Farell y Hersch, 2005; Smith, Smith y Verner, 2006; Rose, 2007; Campbell y Mínguez-Vera, 2008; Adams y Ferreira, 2009). Aunque la evidencia se ha incrementado en los últimos años, sigue siendo escasa.

Por lo que respecta a estudios sobre la presencia de mujeres en cooperativas en España, existe una creciente literatura. La mayoría de los trabajos han sido publicados en revistas de Economía Social, como Ciriec - Revista de Economía Pública, Social y 
Cooperativa o Revesco - Revista de Estudios Cooperativos. A modo de ejemplo, el número 105 (2011) de Revesco es un monográfico sobre "La Economía Social y la Igualdad de Género". No obstante, los estudios que se centran en examinar la participación de mujeres en los órganos de gestión y, más específicamente en el Consejo Rector de las cooperativas, son muy escasos, destacando por su relación con la temática abordada en este estudio, los trabajos de Mateos et al. (2009), Esteban, Gargallo y Pérez (2010; 2013) y Esteban (2012). Sin embargo, que tengamos conocimiento, ningún trabajo examina el efecto de la presencia de mujeres en el Consejo Rector en la rentabilidad y endeudamiento de las cooperativas.

Las sociedades cooperativas constituyen una fuente fundamental de puestos de trabajo y generan espíritu colaborador en la Unión Europea (Chaves y Monzón, 2007), por ello, son vitales para promover la cooperación y el empleo. En este sentido, Diaz y Marcuello (2013) observan que estas organizaciones empleaban en torno al 1,6\% de la población española en 2010, unos 300.000 trabajadores y que el Valor Añadido Bruto que aportaban se situaba en unos 5.200 millones de euros en 2008, un 0,65\% del PIB español. Además, los gobiernos de las cooperativas suelen ser menos estructurados y las actividades llevadas a cabo por sus miembros son variadas, por lo que cada uno de ellos puede tener mayor capacidad para la toma de decisiones. Por tanto, la influencia de la diversidad de género podría ser mayor en este tipo de organizaciones, lo que las hace interesantes para el estudio de la contribución de las mujeres en la administración. Como se ha señalado con anterioridad, la principal contribución de este trabajo es el análisis del impacto de la diversidad de género en el Consejo Rector de las cooperativas en la rentabilidad y el endeudamiento de las mismas.

Los resultados ponen de manifiesto que las cooperativas con mayor representación femenina en su Consejo Rector (equivalente al Consejo de Administración en las sociedades capitalistas) tienden a unos niveles de rentabilidad superiores y un menor ratio de endeudamiento.

\section{TEORÍA E HIPÓTESIS}

Uno de los principales órganos de autoridad o de toma de decisiones en las cooperativas lo constituye la Asamblea General. Se trata de la reunión de la totalidad de los socios, constituida para considerar y adoptar acuerdos sobre asuntos que, legal o reglamentariamente, son de su competencia, y cuyas decisiones son obligatorias para todos los miembros. La Asamblea General es el equivalente a la Junta General de Accionistas en las 
sociedades anónimas, con la diferencia de que el derecho a voto de los accionistas está en función de su participación en el capital social, mientras que entre los cooperativistas rige el principio de un socio, un voto. Otro órgano de toma de decisiones es el Consejo Rector, cuyo equivalente es el Consejo de Administración en las sociedades capitalistas. Al igual que en estas organizaciones, los consejeros son elegidos por los propietarios (socios).

Las cooperativas se caracterizan por ser sociedades de propiedad colectiva donde sus miembros pueden adoptar diferentes roles, propietario, directivo, proveedor, cliente y trabajador; pudiendo incluso adoptar varios de estos roles simultáneamente. El gran poder que los gestores de las cooperativas tienen hace que la teoría de la agencia proporcione un buen marco para el estudio de la relación de sus miembros (Mínguez-Vera, Martín-Ugedo y ArcasLario, 2010). Son, además, organizaciones con una gran resistencia a situaciones de crisis y fuerte arraigo en los municipios, lo que les da una imagen de organizaciones socialmente responsables (Campos-Climent y Chaves-Ávila, 2012). Una de las líneas de las políticas de responsabilidad social empresarial es aprovechar el potencial empresarial mediante la gestión del talento (Server y Capó, 2009), lo que, entre otras cosas, implica buscar a los mejores sin diferencias por género.

La progresiva incorporación de la mujer a la dirección de las empresas, y la proliferación en diversos países, entre ellos España, de leyes de igualdad de género, ha despertado el interés de los investigadores en el estudio de la presencia de la mujer en los Consejos de Administración (Heidrick y Struggles, 2007; Carter et al., 2003; Campbell y Mínguez-Vera, 2008).

En España son varias las medidas para equiparar las oportunidades entre hombres y mujeres en diversos aspectos sociales. Entre ellas, el Código Unificado de Buen Gobierno (2015) que recomienda una discriminación positiva de la mujer para equilibrar la representación de hombres y mujeres en los Consejos de Administración, y que pretende situar en un $30 \%$ la representación femenina en los Consejos de Administración de las empresas con más de 250 trabajadores. Pero estas normas son recomendaciones y se han centrado en grandes empresas capitalistas.

La ACI (Alianza Cooperativa Internacional) establece que las cooperativas deben asegurar la ausencia de barreras para ser socio por razón de género, así como asegurar que las mujeres participan en igualdad numérica en los programas de educación y desarrollo de 
liderazgo. Por estas particularidades, la participación de la mujer en las cooperativas es superior a la media del total de empresas (Mateos et al., 2009). Además, la aplicación de los principios cooperativos establece una serie de valores, como la igualdad, la equidad o la solidaridad, que favorecen la participación de la mujer en los órganos decisionales.

Diversos estudios muestran que un mayor número de mujeres en los Consejos representativos de las cooperativas puede ayudar a que las condiciones de trabajo mejoren y se consiga un equilibrio económico, social y cultural en este tipo de organizaciones (Fregidou, 2004; Gómez, 1998). Además, las cooperativas más jóvenes presentan una mayor participación de mujeres en sus órganos de gobierno, lo que podría ser reflejo de un cambio de tendencia en los aspectos relacionados con la paridad en el ámbito empresarial (Esteban et al., 2010).

En las últimas décadas la preparación de la mujer ha aumentado de forma importante, aunque este incremento no es proporcional a su participación en los puestos directivos de las empresas (Farrell y Hersch, 2005). Esta escasa participación se fundamenta en una barrera que dificulta la promoción laboral femenina llamada "glass ceiling" ("techo de cristal"). Esta barrera viene explicada, entre otros, por la situación familiar que hace que las mujeres tengan una mayor propensión a interrumpir su proyección laboral (Doeringer y Piore, 1971), el papel de la mujer en el sistema capitalista con reducción de la calidad de las condiciones laborales (Engels, 1988) o por los puestos de trabajo considerados tradicionalmente masculinos y femeninos (Benhabib y Cornella, 1990). Es más, hay evidencia de que las mujeres directivas en las cooperativas se centran en sectores tradicionalmente femeninos relacionados con los servicios y en cooperativas de menor tamaño (Fregidou, 2004 - cooperativas suecas y, Berenguer, Cerver, De La Torre y Torcal, 2004 - cooperativas valencianas).

Mientras que las cooperativas y sociedades laborales cumplen con la paridad mínima establecida por la Ley Orgánica 3/2007 (Ley Orgánica 3/2007, de 22 de marzo, para la igualdad efectiva de mujeres y hombres. Publicada en BOE 71 de 23 de Marzo de 2007) respecto al número de mujeres trabajadoras, entorno al 40\%, no ocurre lo mismo en puestos de toma de decisiones donde este porcentaje es mucho más bajo (Martínez, Arcas y García, 2011).

La mayoría de los estudios en gobierno corporativo (Heidrick y Struggles, 2007; Carter et al., 2003; Campbell y Mínguez-Vera, 2008) se han centrado en el enfoque de la 
teoría de la agencia a la hora de estudiar el efecto de la gestión de la mujer. Una "relación de agencia" es un contrato por el que una o más personas (principal/s) encargan a otra/s persona/s (agente/s) la realización de una serie de servicios con delegación en la toma de algunas decisiones (Jensen y Meckling, 1976). Sin embargo, para que los problemas de agencia se materialicen, hace falta no sólo que existan conflictos de objetivos entre principal y agente, sino también que exista asimetría informativa entre ellos.

La mayoría de los estudios coinciden que estos problemas son más complejos en las cooperativas debido a que los socios juegan muy diversos papeles y, además, algunos de ellos asumen más de un rol. De hecho, aunque algunos estudios sugieren que en las cooperativas no existe separación entre propiedad y control y que, en consecuencia, no existen conflictos de objetivos (Hansmann, 1988), otros estudios, como el de Spear (2004), pone el énfasis en la escasa influencia que los socios de las cooperativas ejercen sobre la dirección, hasta el punto de indicar que la discrecionalidad de los directivos en las cooperativas es superior a la de las empresas capitalistas.

A este argumento hay que añadir que, en la actualidad, el número de socios en las cooperativas crece (Morales, 2004), representando distintos roles, lo que conlleva distintos objetivos e intereses (Hansmann, 1988); y, por otro lado, como señala Orellana (2002), en la mayoría de las cooperativas la administración es delegada en los directivos o en agentes profesionales, lo que hace más difícil poder ejercer un control efectivo que permita reducir el conflicto de intereses entre los socios y directivos.

Blau (1977) define la diversidad como "la variedad de atributos individuales dentro de un grupo". Los efectos de la diversidad en el funcionamiento y rendimiento de los grupos de trabajo está caracterizado como "una espada de doble filo" (Milliken y Martins, 1996 y Nielsen y Huse, 2010; entre otros). Es decir, la diversidad tiene efectos positivos y negativos.

La mayoría de los argumentos predicen un efecto positivo de la diversidad en los beneficios de un grupo. Además, Robinson y Dechant (1997) afirman que una mayor diversidad en los grupos de trabajo implica un mayor conocimiento del entorno y una mayor identificación con clientes y empleados, lo que incrementa su habilidad de penetración en el mercado.

De forma similar, cuanto mayor sea la diversidad del grupo, sus miembros tendrán puntos de vista y perspectivas diferentes, así como, más soluciones alternativas a un 
problema, llevando a la adopción de decisiones más realistas (Morrison, 1992). Así, la diversidad incrementaría la creatividad y la innovación y mejoraría la resolución de problemas (Robinson y Dechant, 1997). Milliken y Martins (1996) sugieren que un equipo diversificado tiene acceso a una red de contactos más amplia y a un conjunto más amplio de información, conocimientos y apoyo que caen dentro de dicha red. Además, la comunicación entre el grupo de alta dirección y el resto debería ser más frecuente y de mayor calidad cuando el grupo es diverso (Ancona y Caldwel, 1988).

También existen diversos trabajos que sostienen que la diversidad puede tener efectos nocivos en el rendimiento de un grupo. Sus argumentos se basan el hecho de que los individuos diferentes tienen tendencia a no coincidir en su percepción o ideas (Nielsen y Huse, 2010), sería un grupo menos integrado y más insatisfecho (Milliken y Martins, 1996). Además, los grupos heterogéneos son más proclives a presentar dificultades de comunicación y coordinación y, como consecuencia, hay menos cohesión y relaciones interpersonales satisfactorias (Forbes y Milliken, 1999).

Siguiendo con la diversidad, pero centrándonos en la diversidad de género en las empresas, la presencia de mujeres en los Consejos afecta a la administración de la empresa y, por tanto, puede influir en el valor de la compañía. Sin embargo, la presencia de mujeres en los Consejos también tiene importantes implicaciones desde un punto de vista ético y de cumplimiento de cuotas (Campbell y Mínguez-Vera, 2008; Carter et al., 2010). Aunque las empresas encuadradas dentro de la Economía Social ponderan de manera diferente la importancia de las cuestiones económicas versus las cuestiones sociales y éticas que las empresas capitalistas, ambos tipos de empresas se ven afectadas por ambos aspectos (económicos y éticos).

Como ya se ha mencionado, el Consejo de Administración tiene cuatro funciones: seguimiento y control de los gerentes, proporcionar información y asesoría a los consejeros, supervisar el cumplimiento de leyes y reglamentos aplicables y vincular las corporaciones con el entorno (Mallin, 2004). Las funciones del Consejo han tenido un largo número de clasificaciones (Loden, 1985; Baysinger y Hoskisson, 1990; Nielsen y Huse, 2010; entre otros). Una de esas clasificaciones distingue entre control estratégico y control financiero (Loden, 1985; Baysinger y Hoskisson, 1990). El primero es generalmente a largo plazo y orientado cualitativamente mientras que el segundo es normalmente a corto plazo y orientado cuantitativamente. Loden (1985) apunta que las mujeres están orientadas cualitativamente y 
los hombres cuantitativamente, por tanto, las mujeres son mejores en funciones específicas. Lo que lleva a afirmar que las mujeres influyen positivamente en funciones relacionadas con la responsabilidad social corporativa y el control estratégico. Esta particular orientación lleva a la mujer a proporcionar a los empleados grandes beneficios complementarios como vacaciones, ventajas sociales y seguro médico (Rand y Tarp, 2011).

Además, Hillman, Canella y Harris (2002) afirman que las mujeres tendrían experiencias no tradicionales, y aportan perspectivas, experiencias y estilos de trabajo únicos comparado con los hombres. Además, Daily y Dalton (2003), argumentan que la presencia de la mujer puede mejorar las deliberaciones del Consejo con un estilo de comunicación más participativo y orientado a procesos y que también mejoraría los procesos de toma de decisiones animando a considerar más opciones estratégicas y una variedad de intereses y necesidades del cliente. La presencia femenina mejoraría igualmente la imagen de la compañía y esto tendría un efecto positivo en el comportamiento del cliente (Smith et al., 2006). Finalmente, la mujer introduciría más discusión creativa en el Consejo (Robinson y Dechant, 1997).

Por el contrario, la presencia de la mujer en el Consejo de Administración también tendría influencias negativas, ya que tienen menos relaciones con Consejos de Administración de otras compañías (Zelechowski y Bilimoria, 2004), y es menos probable que tengan espíritu empresarial (Kesner, 1988). También hay autores que apuntan la posibilidad de no influencia de la presencia de la mujer en el Consejo de Administración en el rendimiento de la compañía. Argumentan que las mujeres directivas reniegan de los estereotipos y valores femeninos y, en consecuencia, dicen que tienen un comportamiento similar al de los hombres directivos (Powell, 1990 y Brancato y Patterson, 1999).

La evidencia empírica previa del efecto de la diversidad de género en el rendimiento de la compañía capitalista en los Estados Unidos no es concluyente, pero mayoritariamente apoya una relación positiva. Shrader et al. (1997) y Welbourne, Cycyota y Ferrante (2007) encuentran una relación positiva entre la diversidad de género y diferentes medidas del rendimiento de la compañía. Fenwick y Neal (2001) encuentran que el género está positivamente relacionado con unas medidas del rendimiento pero no hay relación con otras. Harel, Tzafrir y Baruch (2003) también encuentran que las mujeres directivas alcanzan mejores resultados en términos de rendimiento y eficiencia que los hombres. Farell y Hersch (2005) observan que no hay relación entre la diversidad de género y el rendimiento de la 
compañía. Shrader et al. (1997) también encuentra que no hay relación entre la diversidad de género y algunas medidas contables de rendimiento, pero sí hay una relación negativa con otras medidas contables. Finalmente, Jehn y Bezrukova (2004) encuentran una relación negativa entre el porcentaje de mujeres en el Consejo de Administración y varias medidas contables de valor financiero. Adams y Ferreira (2009) también encuentran, por término medio, que el efecto de la diversidad de género en el rendimiento de la compañía es negativo.

La evidencia en Europa tampoco es concluyente. Campbell y Mínguez-Vera (2008) encuentran un efecto positivo de la diversidad de género en el valor de empresas cotizadas. Du Rietz y Henrekso (2000), para una muestra de empresas suecas, y Smith et al. (2006) y Rose (2007), para empresas danesas, no encuentran ninguna influencia de la presencia de la mujer en el Consejo de Administración en el rendimiento. Un resultado similar fue obtenido por Randøy, Thomsen y Oxelheim (2006) para grandes empresas de diferentes países escandinavos (Dinamarca, Noruega y Suecia). Sin embargo, Böhren y Ström (2007) confirman una relación negativa de la presencia de la mujer en Consejos de Administración noruegos y el valor de la empresa.

Siguiendo la mayoría de la evidencia previa que apunta a una influencia positiva de la presencia de la mujer en el Consejo de Administración en el rendimiento de la compañía, se proponen las siguientes hipótesis:

Hipótesis 1.1 (H1.1): Las cooperativas cuyo Consejo Rector tiene una mayor representación femenina presentan mayor rentabilidad económica.

Hipótesis 1.2 (H1.2): Las cooperativas cuyo Consejo Rector tiene una mayor representación femenina presentan mayor rentabilidad financiera.

En el ámbito de la financiación empresarial, la mayoría de la evidencia empírica señala que las empresas gestionadas por mujeres presentan una menor cuantía de endeudamiento (Carter y Shaw, 2006).

La utilización de menor endeudamiento por parte de las mujeres puede atribuirse a sus diferentes ambiciones y objetivos. El Informe del Banco Mundial (2012) muestra que las mujeres suelen ser más prudentes, con menor nivel de ambición. Además, Carter y Shaw (2006) obtienen que la presencia de la mujer en la gestión se asocia a un menor endeudamiento. Incluso, Hernández-Nicolás, Martín-Ugedo y Mínguez-Vera (2015) 
encuentran que la presencia de la mujer en la toma de decisiones tiende a un menor endeudamiento con una deuda de menor coste y mayor plazo, en pequeñas y medianas empresas de nueva creación.

Las diferencias entre las experiencias de hombres y mujeres son fundamentales para dar lugar a formas radicalmente diferentes de ver el mundo (Fischer, Nakakoji, Ostwald, Stahl y Sumner, 1993). Siguiendo este planteamiento, existe un argumento de apoyo adicional que indica que una mayor diversidad de género se asocia con un riesgo inferior. Así, Jianakoplos y Bernasek (1998) sostienen que las mujeres son más reacias al riesgo que los hombres, opinión coincidente con Collerette y Aubry (1990) y Scherr, Sugrue y Ward (1993), entre otros. De esta manera, Smith et al. (2006) sostienen que una mayor proporción de mujeres en tareas decisorias puede afectar el grado de riesgo asumido por la empresa.

Además, Miller, Burker y Glick (1998), entre otros, indican que los miembros de grupos homogéneos de gestión puede tener menos barreras cognitivas e interpersonales que superar para llegar a un acuerdo sobre estrategias agresivas y arriesgadas. Así, los grupos homogéneos tienden a adoptar decisiones de mayor riesgo. Lundeberg, Fox y Punccohar (1994) sugieren que las mujeres son mucho más seguras que los hombres en sus decisiones de inversión. Esta diferencia en la confianza causaría diferencias en el comportamiento arriesgado.

En cuanto a la evidencia empírica, algunos investigadores encontraron que las mujeres son más adversas al riesgo (DiBerardinis, Ramage y Levitt, 1984 y Collerette y Aubry 1990). Olsen y Cox (2001) investigaron las diferencias de género en las actitudes hacia el riesgo para los inversores con formación profesional, obteniendo que en las inversoras ponderan los atributos de riesgo, tales como la posibilidad de la pérdida y la ambigüedad, en mayor medida que sus colegas masculinos. Además, las mujeres tienden a enfatizar la reducción del riesgo más que los hombres en la construcción de una cartera de activos.

La mayor parte de los estudios concluyen que las mujeres empresarias hacen un mayor uso de los recursos propios y menos de la deuda, excepción hecha del dinero prestado por familiares y amigos (Verheul y Thurik, 2001; Orser, Riding y Manley, 2006; Roper y Scott, 2009). 
De acuerdo con la mayoría de los argumentos sobre el bajo endeudamiento de las empresas dirigidas por mujeres y la preferencia de éstas y de los grupos heterogéneos sobre el endeudamiento se formula la siguiente hipótesis:

Hipótesis 2 (H2): Las cooperativas cuyo Consejo Rector tiene una mayor representación femenina presentan menor nivel de endeudamiento.

\section{MUESTRA, DATOS Y METODOLOGÍA}

\subsection{Muestra}

La muestra utilizada ha sido elaborada a partir de la base de datos SABI (Sistema de Análisis de Balances Ibéricos). Esta base de datos dispone de información contable de empresas españolas y portuguesas, obtenida a partir de las Cuentas Anuales depositadas en los registros mercantiles.

La muestra la componen empresas españolas que en 2010 tomaban la forma jurídica de cooperativas ${ }^{3}$. La muestra inicial sin depurar incluye 5.636 cooperativas. De ahí se han eliminado aquellas observaciones en las que se han detectado datos anómalos (por ejemplo, cuando el activo no coincide con el pasivo), así como aquellas cooperativas que no proporcionan información contable de las variables utilizadas en los análisis. De igual modo, se han eliminado los datos que excedían el valor de media más/menos dos veces la desviación típica con el fin de eliminar las observaciones extremas (Levine, Berenson y Krehbiel, 2006). La muestra final está compuesta por un total de 4.750 cooperativas.

\subsection{Datos y Metodología}

Para el análisis de la rentabilidad se han considerado dos variables dependientes: la rentabilidad económica, ROA, calculada como el cociente entre el beneficio antes de intereses e impuestos y el total de activos, y la rentabilidad financiera, ROE, obtenida como el cociente entre el resultado del ejercicio y los recursos propios ${ }^{4}$.

Como medidas de género se han contemplado las siguientes: Una variable, ADM, que mide la proporción de mujeres en el Consejo, calculada como el número de mujeres

\footnotetext{
${ }^{3}$ Los datos fueron recopilados en Mayo de 2013.

${ }^{4}$ Los recursos propios incluyen el capital, las reservas, resultados de ejercicios anteriores, otras aportaciones de los socios, el excedente de la cooperativa (positivo o negativo), Remuneraciones al capital a cuenta y retorno cooperativo a cuenta entregados en el ejercicio, los fondos capitalizado y otros instrumentos del patrimonio neto.
} 
consejeras dividido por el total de componentes del Consejo Rector. Además, se utilizan dos índices para medir la diversidad de género. Por un lado, el índice BLAU definido como: $1-\sum_{i=1}^{n} P_{i}^{2}$, donde Pi hace referencia a la proporción de consejeras. Los valores de este índice oscilan entre 0 y un máximo de 0,5 cuando existe el mismo porcentaje de administradores que de administradoras (Blau, 1977). Por otro, el índice de SHANNON, definido como: - $\sum_{i=1}^{n} P_{i} \ln P_{i}$, donde Pi se calcula de forma similar al utilizado por el índice de Blau. Los valores de este índice oscilan entre 0 y un máximo de 0,69 , siendo esta cifra la que se corresponde con la mayor diversidad posible (Harrison y Klein, 2007).

En cuanto a las variables de control para el análisis de la rentabilidad, se contemplan las siguientes (Waddock y Graves, 1997): el ratio de endeudamiento, END, medido como el cociente entre el total de deuda y el total de activo (Scherr y Hulburt, 2001), el tamaño, TAM, calculado como el logaritmo neperiano del total de activos (Fattouh, Harris y Scaramozzino, 2008) y la edad, EDAD, calculado como el logaritmo neperiano del total de años desde la constitución de la cooperativa.

Los modelos propuestos son los siguientes:

$$
\begin{aligned}
& R O A_{i}=\beta_{0}+\beta_{1} G_{E N}+\beta_{2} E N D_{i}+\beta_{3} \text { TAM }_{i}+\beta_{4} E D A D_{i}+u_{i} \\
& R O E_{i}=\beta_{0}+\beta_{1} G E N_{i}+\beta_{2} E N D_{i}+\beta_{3} T_{A M_{i}}+\beta_{4} E D A D_{i}+u_{i}
\end{aligned}
$$

donde $\mathrm{i}=1, \ldots, \mathrm{n}$, denota la cooperativa y GEN incluye, alternativamente, las variables de género anteriormente expuestas.

Para el análisis del endeudamiento, se utiliza como variable dependiente el ratio de endeudamiento, END, comentado anteriormente.

En cuanto a las variables de control empleadas, son las siguientes: la proporción de activo no corriente, AFIJO, calculada como el cociente entre el activo no corriente y el total de activo (Masayuki, 2012); la rentabilidad económica, ROA; el riesgo económico, RIESGO, obtenido como la diferencia al cuadrado de la rentabilidad económica anual de una cooperativa y la rentabilidad económica anual media de todas las cooperativas, multiplicada por el signo de dicha diferencia (Gaud, Jani, Hoesli y Bender, 2005) y el tamaño, TAM, comentado anteriormente. 
El modelo planteado relativo al endeudamiento es el siguiente:

$$
E N D_{i}=\beta_{0}+\beta_{1} \text { GEN }_{i}+\beta_{2} \text { AFIJO }_{i}+\beta_{3} \text { ROA }_{i}+\beta_{4} \text { RIESGO }_{i}+\beta_{5} \text { TAM }_{i}+u_{i}
$$

donde $\mathrm{i}=1, \ldots, \mathrm{n}$, denota la cooperativa y GEN incluye, alternativamente, las variables de género anteriormente expuestas.

En la tabla 1 se presentan los estadísticos que describen las variables utilizadas en este estudio.

Tabla 1. Estadísticos Descriptivos

\begin{tabular}{lccccc}
\hline Variable & Observaciones & Media & Desv. Típ. & Mínimo & Máximo \\
\hline ADM & 1015 & 0,132 & 0,255 & 0,000 & 1,000 \\
BLAU & 1015 & 0,099 & 0,179 & 0,000 & 0,500 \\
SHANNON & 1015 & 0,145 & 0,257 & 0,000 & 0,690 \\
ROA & 1165 & 0,009 & 3,153 & $-0,108$ & 0,112 \\
ROE & 1165 & 0,025 & 0,103 & $-0,550$ & 0,570 \\
AFIJO & 1118 & 0,605 & 0,489 & 0,000 & 1,000 \\
END & 1196 & 0,530 & 0,271 & 0,000 & 1,000 \\
TAM & 584 & 2,250 & 1,568 & $-4,000$ & 7,000 \\
EDAD & 1160 & 1,443 & 0,447 & 0,630 & 8,937 \\
RIESGO & 4750 & 0,022 & 0,543 & $-0,180$ & 0,180 \\
\hline
\end{tabular}

Variables: ADM (proporción de mujeres en el Consejo); BLAU (índice de diversidad de Blau, proporción de mujeres consejeras); SHANNON (índice de diversidad de Shannon, proporción de mujeres consejeras); ROA (BAII dividido por el total de activo); ROE (resultado del ejercicio dividido por el total de recursos propios); AFIJO (proporción de activo no corriente); END (total de deuda dividido por el total de activo); TAM (logaritmo neperiano del total de activo); EDAD (logaritmo neperiano de los años desde la constitución de la cooperativa); RIESGO (diferencia al cuadrado de la rentabilidad económica anual de una empresa y la rentabilidad económica anual media de todas las empresas, multiplicada por el signo de dicha diferencia).

El porcentaje de mujeres en el Consejo Rector es de un 13,20\%, algo superior al observado en las grandes empresas donde solo alcanzan un valor próximo al 10\% (Carrasco y Laffarga, 2007) y al observado en las Pymes donde tienen una representación de 8,4\% (Martín-Ugedo y Mínguez-Vera, 2014).

En cuanto a las cifras aportadas por otros estudios centrados en las cooperativas españolas, los valores varían dependiendo del territorio y del sector de actividad. Por ejemplo, en las cooperativas de trabajo asociado de Madrid se observa un 35\% de consejeras (Fernández, 2000). En el mismo sector pero en la Comunidad Valenciana el porcentaje si sitúa en un 27\% (Berenguer, Castellví, Cerver, Juan, Torcal, De La Torre, 1999). En el país 
vasco, en general, la presencia de la mujer en los órganos de gobierno se sitúa en el $20 \%$ (CSCE, 2001), mientras que en Mondragón Corporación Cooperativa (MCC) las mujeres ocupaban un 16\% de los cargos en los Consejos Rectores en el año 2000 (Elio, 2006). Finalmente, Esteban et al. (2013) muestran una cifra del 20,9\% para una muestra de cooperativas agrícolas turolenses. Sin embargo, están cifras siguen siendo bajas ya no cumplen la recomendación de la Unión Europea del 40\%, considerado como valor que asegura una representación paritaria. Este objetivo si se alcanza, por ejemplo, en las cooperativas suecas, cuyo porcentaje de participación el Consejo Rector se situaba en un $41 \%$ en 2004 (Fregido, 2004).

La rentabilidad económica presenta una media del 0,93\%, mientras que la rentabilidad financiera se sitúa en el 2,50\%. En cuanto al nivel de deuda, éste representa el 53,00\%.

La metodología utilizada es la de Mínimos Cuadrados en Tres Etapas, más eficiente que la de Mínimos Cuadrados en 2 Etapas y la de Mínimos Cuadrados Ordinarios (Abou y Abdelfattar, 2014). Dicha metodología se ha usado por la peculiaridad de la muestra, compuesta por empresas cuya forma jurídica es la Cooperativa con datos sólo para el año 2.010. La circunstancia de que sólo se puede obtener datos para la identificación de la presencia de la mujer en órganos de decisión en la base de datos SABI para un año, imposibilita la utilización de una metodología más potente como la de datos de panel.

\section{RESULTADOS}

Los resultados de la estimación del análisis de la influencia del género sobre la rentabilidad económica y la rentabilidad financiera se presentan en las tablas 2 y 3 . 
Tabla 2. Estimación Influencia del Género sobre ROA

\begin{tabular}{|c|c|c|c|}
\hline Modelo & $\mathrm{ADM}$ & BLAU & SHANNON \\
\hline Constante & $\begin{array}{c}0,560 \\
(0,470)\end{array}$ & $\begin{array}{c}-0,107 \\
(-0,090)\end{array}$ & $\begin{array}{c}-0,111 \\
(-0,100)\end{array}$ \\
\hline GEN & $\begin{array}{c}1,728 * * * \\
(2,810)\end{array}$ & $\begin{array}{c}2,490 * * * \\
(3,010)\end{array}$ & $\begin{array}{c}1,700 * * * \\
(2,980)\end{array}$ \\
\hline END & $\begin{array}{c}0,260 \\
(1,240)\end{array}$ & $\begin{array}{l}0,039^{*} \\
(1,900)\end{array}$ & $\begin{array}{c}0,039 * * \\
(1,920)\end{array}$ \\
\hline TAM & $\begin{array}{c}-0,147 \\
(-1,420)\end{array}$ & $\begin{array}{c}-0,163 \\
(-1,550)\end{array}$ & $\begin{array}{c}-0,164 \\
(-1,560)\end{array}$ \\
\hline EDAD & $\begin{array}{c}-0,295 \\
(-1,100)\end{array}$ & $\begin{array}{c}-0,342 \\
(-1,260)\end{array}$ & $\begin{array}{c}-0,347 \\
(1,280)\end{array}$ \\
\hline $\mathrm{X} 2$ & $10,56 * *$ & $14,51 * * *$ & 14,36 *** \\
\hline
\end{tabular}

$*, * *, * * *$ Significativo al $10 \%, 5 \%$ y $1 \%$, respectivamente.

Variables: GEN (Variable de género, incluye: ADM (proporción de mujeres en el Consejo); BLAU (índice de diversidad de Blau, proporción de mujeres consejeras); SHANNON (índice de diversidad de Shannon, proporción de mujeres consejeras); END (total de deuda dividido por el total de activo); TAM (logaritmo neperiano del total de activo); EDAD (logaritmo neperiano de los años desde la constitución de la cooperativa). Z-Estadístico entre paréntesis. X2: Test de significatividad conjunta.

Concretamente se observa que el género de los componentes del Consejo Rector afecta a la rentabilidad económica y financiera de la Cooperativa, donde se constata una influencia positiva y altamente significativa cuando el consejero es mujer o hay diversidad, representado por las medidas de Blau y Shannon, en la composición de dicho Consejo Rector. Estos resultados están en la línea de los aportados por Smith et al. (2006), Carter et al. (2003) y Welbourne et al. (2007), entre otros, para sociedades capitalistas. Por tanto, la presencia de mujeres en el Consejo Rector parece que reduce el conflicto de intereses y, por tanto, se cumplen la Hipótesis 1.1 (H1.1): Las cooperativas cuyo Consejo Rector tiene una mayor representación femenina presentan mayor rentabilidad económica, y la Hipótesis 1.2 (H1.2): Las cooperativas cuyo Consejo Rector tiene una mayor representación femenina presentan mayor rentabilidad financiera. 
Tabla 3. Estimación Influencia del Género sobre ROE

\begin{tabular}{|c|c|c|c|}
\hline Modelo & ADM & BLAU & SHANNON \\
\hline Constante & $\begin{array}{c}-5,514 * * * \\
(-1,390)\end{array}$ & $\begin{array}{c}-7,501 * * \\
(-1,950)\end{array}$ & $\begin{array}{c}-7,481 * * \\
(-1,950)\end{array}$ \\
\hline GEN & $\begin{array}{l}3,313^{*} \\
(1,640)\end{array}$ & $\begin{array}{c}6,718 * * * \\
(2,490)\end{array}$ & $\begin{array}{c}4,468 * * * \\
(2,390)\end{array}$ \\
\hline END & $\begin{array}{c}0,227 * * * \\
(3,300)\end{array}$ & $\begin{array}{c}0,260 * * * \\
(3,850)\end{array}$ & $\begin{array}{c}0,261 * * * \\
(3,850)\end{array}$ \\
\hline TAM & $\begin{array}{l}-0,632^{*} \\
(-1,850)\end{array}$ & $\begin{array}{c}-0,662 * * \\
(-1,910)\end{array}$ & $\begin{array}{c}-0,667 * * \\
(-1,930)\end{array}$ \\
\hline EDAD & $\begin{array}{c}-1,769 * * \\
(-2,020)\end{array}$ & $\begin{array}{c}-1,854 * * \\
(-2,090)\end{array}$ & $\begin{array}{c}-1,869 * * \\
(-2,110)\end{array}$ \\
\hline $\mathrm{X} 2$ & $15,17 * * *$ & $23,74 * * *$ & $23,38 * * *$ \\
\hline
\end{tabular}

En cuanto a las variables de control, las cooperativas de menor tamaño tienen una rentabilidad financiera mayor. Este resultado viene justificado por ventajas competitivas que presentan las cooperativas como la flexibilidad en la producción (Fariñas y Martín, 2011) y la proximidad a la demanda y la concienciación de las necesidades del consumidor (Vossen, 1998). Sin embargo, el tamaño no afecta a la rentabilidad económica. Si nos centramos en la antigüedad, los resultados determinan que a menos edad, las cooperativas presentan mayor rentabilidad financiera, evidencia que se apoya en que las empresas más viejas tienen a la inercia y la burocratización, por lo que es probable que no presenten la suficiente flexibilidad para adaptarse a los entornos cambiantes (Majumdar, 1997). Sin embargo, la antigüedad no afecta a la rentabilidad económica.

Según los resultados, el nivel de deuda de la cooperativa influye, en general, positivamente tanto en la rentabilidad económica como en la rentabilidad financiera. La deuda la utilizarían para conseguir ventajas fiscales (Pettit y Singer, 1985), que unido a una buena gestión lleva a una mayor rentabilidad para los socios manifestada a través de la rentabilidad financiera y por extensión, esa buena gestión puede tener su reflejo en una mayor rentabilidad económica. 
En la Tabla 4 se presentan los resultados relativos al análisis de la influencia del género sobre el nivel de endeudamiento de la cooperativa. Se observa que un mayor porcentaje de mujeres en el Consejo Rector se relaciona con un menor nivel de endeudamiento. Esto puede venir explicado por la aversión al riesgo de la mujer (Orser et al, 2006; Borghans, Golsten, Heckman y Meijers, 2009) cuya gestión genera decisiones menos arriesgadas (Scherr et al, 1993; Jianakoplos y Bernasek, 1998). Se cumple, por tanto, la Hipótesis 2 (H2): Las cooperativas cuyo Consejo Rector tiene una mayor representación femenina presentan menor nivel de endeudamiento.

En cuanto a las variables de control, se obtienen los siguientes resultados: Un efecto positivo del tamaño y de la rentabilidad económica sobre el ratio de endeudamiento. El mayor tamaño de las empresas genera confianza en el mercado facilitando el acceso a fuentes de financiación externa (Hall, Hutchinson y Michaelas, 2000), por otra parte, las empresas con una mayor rentabilidad económica se les considera más solventes lo que facilita el acceso al endeudamiento (Verheul y Thurik, 2001).

Por otra parte, los resultados muestran que la proporción de activo no corriente presenta un efecto negativo sobre el nivel de endeudamiento. Como ya se ha mencionado, la deuda puede ser utilizada para asegurar ventajas fiscales (Pettit y Singer, 1985), pero también, hay otras deducciones fiscales como la depreciación del activo no corriente (DeAngelo y Masulis, 1980) que podría llevar a una relación negativa entre la proporción de activo no corriente y el nivel de endeudamiento como se refleja en la tabla 4. Además, en los resultados se observa que el nivel de riesgo económico no tiene efecto en el nivel de endeudamiento. 
Tabla 4. Estimación Influencia Género sobre el Nivel de Endeudamiento

\begin{tabular}{|c|c|c|c|}
\hline Modelo & ADM & BLAU & SHANNON \\
\hline Constante & $\begin{array}{c}54,933 * * * \\
(16,860)\end{array}$ & $\begin{array}{c}53,358 * * * \\
(16,280)\end{array}$ & $\begin{array}{c}53,310 \\
(16,260)\end{array}$ \\
\hline GEN & $\begin{array}{c}-7,517 * * \\
(-1,990)\end{array}$ & $\begin{array}{c}3,347 \\
(0,620)\end{array}$ & $\begin{array}{c}2,546 \\
(0,680)\end{array}$ \\
\hline AFIJO & $\begin{array}{c}-8,555^{* * * *} \\
(-4,130)\end{array}$ & $\begin{array}{c}-8,328 * * * \\
(-4,010)\end{array}$ & $\begin{array}{c}-8,331 * * * \\
(-4,020)\end{array}$ \\
\hline ROA & $\begin{array}{c}-2,060 * * * \\
(-8,660)\end{array}$ & $\begin{array}{c}-2,122 * * * \\
(-8,910)\end{array}$ & $\begin{array}{c}-2,123 * * * \\
(-8,920)\end{array}$ \\
\hline RIESGO & $\begin{array}{c}0,035 * * * \\
(5,030)\end{array}$ & $\begin{array}{c}0,036 * * * \\
(5,130)\end{array}$ & $\begin{array}{c}0,036 * * * \\
(5,130)\end{array}$ \\
\hline TAM & $\begin{array}{c}2,500 * * * \\
(4,040)\end{array}$ & $\begin{array}{c}2,646 * * * \\
(4,250)\end{array}$ & $\begin{array}{c}2,651 * * * \\
(4,260)\end{array}$ \\
\hline $\mathrm{X} 2$ & $24,84 * * *$ & $24,10 * * *$ & $24,12 * * *$ \\
\hline \multicolumn{4}{|c|}{$\begin{array}{l}\text { *,**,*** Significativo al } 10 \%, 5 \% \text { y } 1 \% \text {, respectivamente. } \\
\text { Variables: GEN (Variable de género, incluye: ADM (proporción de mujeres en el Consejo); BLAU (índice } \\
\text { de diversidad de Blau, proporción de mujeres consejeras); SHANNON (índice de diversidad de Shannon, } \\
\text { proporción de mujeres consejeras); AFIJO (proporción de activo no corriente); ROA (BAII dividido por el } \\
\text { total de activo); RIESGO (diferencia al cuadrado de la rentabilidad económica anual de una empresa y la } \\
\text { rentabilidad económica anual media de todas las empresas, multiplicada por el signo de dicha diferencia); } \\
\text { TAM (logaritmo neperiano del total de activo). Z-Estadístico entre paréntesis. X2: Test de significatividad } \\
\text { conjunta. }\end{array}$} \\
\hline
\end{tabular}

\section{CONCLUSIONES}

El género y su influencia sobre diversas variables de la empresa han ido captando la atención de los investigadores. Varios han sido los trabajos que han puesto de manifiesto las diferencias, en cuanto a la forma de dirigir una empresa, entre hombres y mujeres, y la repercusión que supone la diversidad de género en los grupos de trabajo (Heidrick y Struggles, 2007; Carter et al., 2003; Campbell y Mínguez-Vera, 2008; entre otros). Sin embargo, son escasos los estudios que se han centrado en la participación de la mujer en las entidades de Economía Social (Berenguer et al., 1999; Fernández, 2000; Freguidou, 2004; Elio, 2006; Esteban et al. 2013), ámbito dónde España es pionera en legislación. Estos estudios de Economía Social se han centrado, en su mayoría, en la gestión del desempeño y la calidad de servicio prestado. Sin embargo, no se ha prestado la suficiente atención a la influencia del género en la gestión empresarial.

La actualidad de los estudios sobre género se ha visto incrementada por el surgimiento de leyes y debates sobre igualdad de género en numerosos países, entre ellos España. Son varias las medidas tomadas en España para equiparar las oportunidades entre hombres y 
mujeres en diversos aspectos sociales. Entre ellas, la Ley de Igualdad de 2007, cuyo objeto es hacer efectivo el derecho de igualdad de trato y oportunidades entre mujeres y hombres, en cualesquiera ámbitos de la vida y, singularmente, en las esferas política, civil, laboral, económica, social y cultural.

En el ámbito de la Economía Social, y concretamente, de las Cooperativas, la igualdad de oportunidades es uno de sus objetivos, que unido al estilo de liderazgo femenino hace interesante este análisis.

Este artículo estudia la influencia de la diversidad de género en la gestión empresarial; concretamente en la rentabilidad y en el nivel de endeudamiento de las cooperativas, empleando para ello información contable del año 2010. El estudio se centra en el contexto español, caracterizado por ser España país pionero en contar con una legislación específica sobre Economía Social (Ley 5/2011, de 29 de marzo).

Se observa que la presencia de la mujer en posiciones de toma de decisiones incrementa la rentabilidad de la cooperativa. Una mayor diversidad lleva a un incremento de la ventaja competitiva de la compañía, incrementa la innovación y puede favorecer tareas relacionadas con la responsabilidad social corporativa. Los resultados también muestran que la presencia de mujeres en el Consejo Rector reduce el nivel de endeudamiento, lo que está en línea con el carácter más prudente de la mujer que señala el Informe del Banco Mundial 2012. También puede deberse a que las mujeres ponderan, en mayor medida, la posibilidad de la pérdida y enfatizan la reducción del riesgo. Por tanto, el establecimiento de cuotas en la dirección de las empresas, no sólo capitalistas sino también relativas a la economía social, no sólo se justifica desde el punto de vista de equidad y justicia social, sino también económico.

Las principales limitaciones de este trabajo es la utilización de un solo año como muestra, al no disponer de datos suficientes para elaborar un panel con que imposibilita el control de la heterogeneidad inobservable de las entidades estudiadas. Por otro lado, se centra únicamente en los objetivos económicos de la cooperativa sin hacer referencia a los aspectos éticos o sociales ya que se carece de los datos necesarios para realizar dicho análisis. En cuanto a las futuras líneas de investigación, sería interesante captar los reseñados objetivos sociales y éticos a través de encuestas personales a los miembros de las cooperativas y ampliar el estudio a cooperativas de otros países. 


\section{BIBLIOGRAFÍA}

ABOU ALI, H. y ABDELFATTAR, Y.M. (2014) Modeling the relationship between natural resource abundance, economic growth and the environment: A cross country study. En: HADRI, K.; MIHAIK, W. Econometric Methods and their application in finance, macro and related fields. Singapur: World Scientific Publishing, pp. 489-510.

ADAMS, R. y FERREIRA, D (2009) Women on the boardroom and their impact on governance and performance. Journal of Financial Economics, vol. 94, № 1, pp. 291-309.

ALONSO, F. (1984) Las relaciones laborales en las cooperativas en España. Revista Española Derecho del Trabajo, Civitas, № 20, pp. 525-560.

ÁLVAREZ, M.B.; LOPEZ, P. y CANTÓ, C. (2000) Las cooperativas en el marco de la teoría de la agencia. Ciriec - Revista de Economía Pública, Social y Cooperativa, No 34, pp. 169-188.

ANCONA, D. y CALDWEL, D. (1988) Beyond task and maintenance: Defining external functions in groups. Group and Organization Studies, vol. 13, № 4, pp. 468-494.

BAYSINGER, B. y HOSKISSON, R. (1990) The composition of boards of directors and strategic control: Effects on corporate strategy. Academy of Management Review, vol. 15, No 1, pp. 72-87.

BENHABIB, S. y CORNELLA, D. (1990) Teoría feminista y teoría crítica. Valencia: Ediciones Alfons el Magnanim, Institució Valenciana D’estudie e investigación, España.

BERENGUER, G.; CASTELlVÍ, J.M.; CERVER, E.; JUAN, C.; TORCAL, R. y DE LA TORRE, A. (1999) El Laberinto de Cristal. Valencia: FVECTA.

BERENGUER, G.; CERVER, E.; De La TORRE, A. y TORCAL, V. (2004) El estilo directivo de las mujeres y su influencia sobre la gestión del equipo de trabajo en las cooperativas valencianas. Ciriec - Revista de Economía Pública, Social y Cooperativa, № 50, pp. 123-149.

BILIMORIA, D. y PIDERIT, S. (1994) Board committee membership: effect of sex -based bias, Academy of Management Journal, vol. 37, № 6, pp. 1453-1477.

BLAU, P. (1977) Inequality and heterogeneity: A primitive theory of social structure. New York: The Free Press.

BÖHREN, Ø. y STRÖM, R. (2007) Aligned, informed, and decisive: characteristics of valuecreating boards, EFA 2007 Ljubljana Meetings Paper. 
BORGHANS, L.; GOLSTEN, B.; HECKMAN, J. y MEIJERS, H. (2009) Gender differences in risk aversion and ambiguity aversion. Journal of the European Economic Association, vol. 7, $\mathrm{N}^{\mathrm{o}} 2-3$, pp. 649-658.

BRANCATO, C. y PATTERSON, J. (1999) Board diversity in U.S. corporations: Best practices for broadening the profile of corporate boards, Research Report 1230-99-RR: The Conference Board.

CAMPBELL, K. y MINGUEZ-VERA, A. (2008) Gender diversity in the boardroom and firm financial performance. Journal of Business Ethics, vol. 83, nº 3, pp. 435-451.

CAMPOS-CLIMENT, V. y CHAVES-ÁVILA, R. (2012) El papel de las cooperativas en la crisis agraria. Estudio empírico aplicado a la agricultura mediterránea española. Cuadernos de Desarrollo Rural, vol. 9, № 69, pp. 175-194.

CARRASCO, A. y LAFFARGA, J. (2007) La diversidad de género en el código unificado español y en la práctica empresarial. Pecnia, $\mathrm{n}^{\circ} 4$, pp. 1-25.

CARTER, D.; D'SOUZA, F.; SIMKINS, B. y SIMPSON, W. (2010) The gender and the ethnic diversity of US boards and board committees and firm financial performance. Corporate Governance: An International Review, vol. 18, № 5, pp. 396-414.

CARTER, S. y SHAW, E. (2006) Women's business ownership: Recent research and policy developments. Report to the Small Business Service. DTI.

CARTER, D.; SIMKIN, B. y SIMPSON, W. (2003) The corporate governance, board diversity, and firm value. The Financial Review, vol. 38, No 1, pp. 33-53.

CHAVES, R. y MONZÓN, J.L. (2007) La Economía Social en la Unión Europea. Bruselas: Ciriec - Comité Económico y Social Europeo, 131, Disponible desde: www.eesc.europa.eu/groups/3/index_en.asp?id=1405GR03EN.

CÓDIGO UNIFICADO DE BUEN GOBIERNO (2015) Acuerdo del Consejo de la Comisión Nacional del Mercado de Valores de 18 de febrero de 2015. Madrid: CNMV.

COLLERETTE, P. y AUBRY, P. (1990) Socio-economic evolution of women business owners in Quebec. Journal of Business Ethics, vol. 9, No 4-5, pp. 417-22.

CSCE (2001) Sensibilidad y Buenas Prácticas de las Cooperativas de la CAPV en el Fomento de Igualdad de Oportunidades entre Hombres y Mujeres. Anuario del Consejo Superior de Cooperativas de Euskadi Año 2000. Vitoria: Consejo Superior de Cooperativas de Euskadi.

DAILY, C. y DALTON, D. (2003) Women in the boardroom: A business imperative. Journal of Business Strategy, vol. 24, № 5, pp. 8-9. 
DeANGELO, H. y MASULIS, R. (1980) Optimal capital structure under corporate and personal taxation. Journal of Financial Economics, vol. 8, № 1, pp. 3-29.

DIAZ FONCEA, M. y MARCUELLO, C. (2013) Evolución del sector cooperativo en España. Artículo presentado en la 4th International CIRIEC Research Conference on the Social Economy, Antwerp (Belgica).

DIBERARDINIS, J.; RAMAGE, K. y LEVITT, S. (1984) Risky shift and gender of the advocate: Information theory versus normative theory. Group and Organisation Studies, vol. 9, $\mathrm{N}^{\mathrm{o}}$ 2, pp. 189-200.

DOERINGER, P. y PIORE, M. (1971) Internal markets and manpower analysis. Lexington: Heath Lexington Books and D.C. Heath and Company.

DU RIETZ, A. y HENREKSO, M. (2000) Testing the female underperformance hypothesis. Small Business Economics, vol. 14, N 1, pp. 1-10.

ELIO CEMBORAIN, E. (2006) Responsabilidad social en las cooperativas: igualdad de oportunidades entre hombres y mujeres. GEZKI, No. 2, pp. 35-71.

ENGELS, F. (1988) El origen de la familia, la propiedad privada y el estado. Debarris: Barcelona.

ESTEBAN SALVADOR, M.L. (2012) El papel de la mujer en las cooperativas agrícolas turolenses. Ciriec - Revista de Economía Pública, Social y Cooperativa, No 74, pp. 177205.

ESTEBAN SALVADOR, M.L.; GARGAllo CASTEL, A. y PÉREZ SANZ, F.J. (2010) Composición del Consejo Rector y género en las cooperativas turolenses. REVESCO. Revista de Estudios Cooperativos, № 101, pp. 7-27.

ESTEBAN SALVADOR, M.L.; GARGALlO CASTEL, A. y PÉREZ SANZ, F.J. (2013) ¿Son las cooperativas más favorables a la presencia de mujeres en los consejos que otras entidades? REVESCO. Revista de Estudios Cooperativos, $\mathrm{N}^{\circ} 110$, pp. 96-128.

FARIÑAS, J.C. y MARTÍN, A. (2001) Tamaño empresarial, flexibilidad de costes y cambio tecnológico. Papeles de Economía Española, № 89-90, pp. 272-285.

FARRELL, K. y HERSCH, P. (2005) Additions to corporate boards: the effect of gender. Journal of Corporate Finance, vol. 11, № 1, pp. 85-106.

FATTOUH, B.; HARRIS, L. y SCARAMOZZINO, P. (2008) Non-linearity in the determinants of capital structure: Evidence from UK firms. Empirical Economics, vol. 34, No 3, pp. 417-438.

FENWICK, G. y NEAL, D. (2001) Effect of gender composition on group performance, Gender. Work y Organization, vol. 8, № 2, pp. 205-225. 
FERNÁNDEZ PLAZA, A. (2000) Investigación sobre mujeres y cooperativismo en la Comunidad de Madrid. Cuadernos Mujer y Cooperativismo, №. 2, pp. 26-27.

FISCHER, G.; NAKAKOJI, K.; OSTWALD, J.; STAHL, G y SUMNER, T. (1993) Embedding critics in design environments. The Knowledge Engineering Review, vol. 8, $\mathrm{N}^{\mathrm{o}}$ 4, pp. 285-307.

FORBES, D. y MILLIKEN, F. (1999) Cognition and corporate governance: Understanding boards of directors as strategic decision-making groups. Academy of Management Review, vol. 24, No 3, pp. 489-505.

FREGIDOU, M. (2004) ¿Es necesario equilibrar el poder en las cooperativas entre mujeres y hombres? Ciriec - Revista de Economía Pública, Social y Cooperativa, № 50, pp. 151163.

GARCÍA, M. (2010) La necesaria armonización internacional del derecho cooperativo: el Caso español. REVESCO. Revista de Estudios Cooperativos, № 102, pp. 79-108.

GAUD, P.; JANI, E.; HOESLI, M. y BENDER, A. (2005) The capital structure of Swiss companies: an empirical analysis using dynamic panel data. European Financial Management, vol. 11, № 1, pp. 51-63.

GÓMEZ, L. (1998) Women in co-operatives: The policy of the International Cooperative Alliance. Journal of Co-operative Studies, vol. 31, pp. 2.

GROSVOLD, J. y BRAMMER, S. (2011) National institutional systems as antecedents of female board representation: An empirical study. Corporate Governance: An International Review, vol. 19, № 2, pp. 116-135.

HALL, G.; HUTCHINSON, P. y MICHAELAS, N. (2000) Industry effects on the determinants of unquoted SMEs' capital structure. International Journal of the Economics of Business, vol. 7, $\mathrm{N}^{\circ} 3$, pp. 297-312.

HANSMANN, H. (1988) Ownership of the firm. Journal of Law, Economy and Organization, pp. 267-304.

HAREL, G.; TZAFRIR, S. y BARUCH, Y. (2003) Achieving organizational effectiveness through promotion of women into managerial positions: HRM practice focus. International Journal of Human Resource Management, vol. 14, № 2, pp. 247-263.

HARRISON, D. y KLEIN, K. (2007) What's the difference? Diversity constructs as separation, variety, or disparity in organization. Academy of Management Review, vol. 32, $\mathrm{N}^{\mathrm{o}}$ 4, pp. 1199-1228.

HEIDRICK y STRUGGLES (2007) Corporate governance in Europe: 2007 Report. Paris, Business Strategy Series. 
HEIDRICK y STRUGGLES. (2011) Challenging board performance. European Corporate Governance Report 2011 [online], [Citado el 20 de Agosto de 2014]. Disponible en: http://www.heidrick.com/PublicationsReports/PublicationsReports/HS_EuropeanCorpGov Rpt2011.pdf.

HERNÁNDEZ-NICOLÁS, C.M.; MARTÍN-UGEDO, J.F. y MÍNGUEZ-VERA, A. (2015)

The influence of gender on financial decisions: Evidence from small start-up firms in Spain. E\&M Economics and Management, vol. 18, № 4, pp. 93-107.

HERRERA, J. y SANCHÍS, R. (1993) Hacia una dirección estratégica de los recursos humanos en las empresas cooperativas. Ciriec - Revista de Economía Pública, Social y Cooperativa, № 14 , pp. 79-103.

HILLMAN, A.; CANNELLA, A. y HARRIS, I. (2002) Women and racial minorities in the boardroom: how do directors differ? Journal of Management, vol. 28, № 6, pp. 747-63.

HUSE, M.; NIELSEN, S. y HAGEN, I. (2009) Women and Employee-Elected Board Members, and Their Contributions to Board Control Tasks. Journal of Business Ethics, vol. 89 , No 1 , pp. 581-97.

INFORME DEL BANCO MUNDIAL. (2012) Washington: World Bank. Disponible en: http://www.bancomundial.org/content/dam/Worldbank/document/PLBSummer12latest.pd f.

JEHN, K.A. y BEZRUKOVA, K. (2004) A field study of group diversity, group context, and performance. Journal of Organizational Behavior, vol. 25, No 6, pp. 1-27.

JENSEN, M. y MECKLING, W. (1976) Theory of the firm: managerial behavior, agency costs and ownership structure. Journal of Financial Economics, vol. 3, pp. 305-360.

JIANAKOPLOS, N. y BERNASEK, A. (1998) Are women more risk averse? Economic Inquiry, vol. 36, $\mathrm{N}^{\mathrm{o}} 4$, pp. 620-630.

KESNER, I. F. (1988) Directors' characteristics and committee membership: an investigation of type, occupation, tenure and gender. Academy of Management Journal, vol. 31, $\mathrm{N}^{\mathrm{o}} 1$, pp. 66-84.

LEY 5/2011, de 29 de marzo, de Economía Social. BOE núm. 76 de 30 de Marzo de 2011.

LEY ORGÁNICA 3/2007, de 22 de marzo, para la igualdad efectiva de mujeres y hombres. BOE 71 de 23 de Marzo de 2007.

LEY 27/1999, de 16 de julio, de Cooperativas. BOE núm. 170 de 17 de Julio de 1999.

LEVINE, D. M.; BERENSON, M. L. Y KREHBIEL, T.C. (2006) Estadística para administración. Madrid: Pearson Educación. 
LODEN, M. (1985) Feminine leadership, or how to succeed in business without being one of the boy. New York: Times Books.

LUNDEBERG, M.; FOX, P. y PUNCCOHAR, J. (1994) Highly confident but wrong: gender differences and similarities in confidence judgments. Journal of Educational Psychology, vol. 86, No 1 , pp. 114-121.

MAJUMDAR, S. (1997) The impact of size and age on firm-level performance: some evidence from India. Review of Industrial Organization, vol. 12, No 2, pp. 231-241.

MALLIN, C. (2004) Corporate governance. Boston: Harvard University Press.

MARTÍN-UGEDO, J.F. y MÍNGUEZ-VERA, A. (2014) Firm performance and women on the board: Evidence from Spanish small and medium-sized enterprises. Feminist Economics, vol. 20, №3, pp. 136-162.

MARTÍNEZ, I.M.; ARCAS, N. y GARCÍA, M. (2011) La influencia del género sobre la responsabilidad social empresarial en las entidades de economía social. REVESCO. Revista de Estudios Cooperativos, № 105, pp. 143-172.

MASAYUKI, M. (2012) Financial constraints in intangible investments: Evidence from Japanese firms, RIETI Discussion Paper 12-E-045.

MATEOS, R.; ESCOT, L. y GIMENO, R. (2006) Análisis de la presencia de la mujer en los Consejos de Administración de las mil mayores empresas españolas. Fundación de las Cajas de Ahorros, Documento de trabajo 263.

MATEOS, R.; ESCOT, L. y GIMENO, R. (2010) Discriminación en los Consejos españoles: análisis e implicaciones económicas. Revista de Economía Aplicada, vol. 18, No 53, pp. 131-162.

MATEOS, R.; GIMENO, R. y ESCOT, L. y (2011) Disentangling discrimination on Spanish boards of directors. Corporate Governance: An International Review, vol. 19, № 1, pp. 77-95.

MATEOS, R.; ITURROIZ, J. y GIMENO, R. (2009) La participación financiera y el papel de la mujer en la toma de decisiones de las sociedades cooperativas: los Consejos de Administración. Revista Europea de Dirección y Economía de la Empresa, vol. 18, № 3, pp. 65-82.

MILLER, C.; BURKER, L. y GLICK, W. (1998) Cognitive diversity among upper-echelon executives: Implications for strategic decision processes. Strategic Management Journal, vol. 19, $\mathrm{N}^{\circ} 1$, pp. 39-58. 
MILLIKEN, F. y MARTINS, L. (1996) Searching for common threads: Understanding the multiple effects of diversity in organizational groups. Academy of Management Journal, vol. 21, No 2, pp. 402-433.

MÍNGUEZ-VERA, A.; MARTÍN-UGEDO, J.F. y ARCAS-LARIO, N. (2010) Agency and property rights theories in agricultural cooperatives: Evidence from Spain. Spanish Journal of Agricultural Research, vol. 8, No 4, pp. 908-924.

MORALES, A. (2004) La dirección de la empresa de trabajo asociado: Una revisión de los estudios empíricos. Revista de Economía Pública, Social y Cooperativa, vol. 48, pp. 99122.

MORRISON, A. (1992) The new leaders: Guidelines on leadership diversity in America. San Francisco: Jossey Bass.

NIELSEN, S. y HUSE, M. (2010) Women director's contribution to board decision-making and strategic involvement: The role of equality perception. European Management Review, vol. 7, $\mathrm{N}^{\circ}$ 1, pp. 16-29.

NILSSON, J. (1996) The nature of values and principles. Annals of public and cooperative economics, vol. 67, $\mathrm{N}^{\mathrm{o}} 4$, pp. 633-653.

OLSEN, R y COX, C. (2001) The influence of gender on the perception and response to investment risk: The case of professional investors. Journal of Psychology and Financial Markets, vol. 2, № 1, pp. 29-36.

ORELLANA, W.E. (2002) El control de las empresas de trabajo asociado: Revisión crítica desde una perspectiva de la agencia, de los principales mecanismos de control y análisis de sus repercusiones organizativas. Revista de Economía Pública, Social y Cooperativa, $\mathrm{N}^{\circ}$ 42, pp. 49-80.

ORSER, B.; RIDING, A. y MANLEY, K. (2006) Women entrepreneurs and financial capital. Entrepreneurship Theory and Practice, vol. 30, № 5, pp. 643-665.

PETTIT, R. y SINGER, R. (1985) Small business finance: a research agenda. Financial Management, vol. 14, № 3, pp. 47-60.

POWELL, G. (1990) One more time: Do female and male managers differ? The Executive, vol. 4, No 3, pp. 68-75.

RAND, J. y TARP, F. (2011) Does gender influence the provision of fringe benefits? Evidence from Vietnamese SMEs. Feminist Economics, vol. 17, № 1, pp. 59-87.

RANDØY, T.; THOMSEN, S. y OXELHEIM, L. (2006) A Nordic perspective on corporate board diversity. Artículo presentado en la European International Business Academy Annual Conference, Fribourg. 
ROBINSON, G. y DECHANT, K. (1997) Building a business case for diversity. Academy of Management Executive, vol. 11, № 3, pp. 21-30.

ROPER, S y SCOTT, J. (2009) Perceived financial barriers and the start-up decision. International Small Business Journal, vol. 27, № 2, pp. 149-171.

ROSE, C. (2007) Does female board representation influence firm performance? The Danish evidence. Corporate Governance: An International Review, vol. 15, № 2, pp. 404-413.

SCHERR, F. y HULBURT, H. (2001) The debt maturity structure of small firms. Financial Management, vol. 30, pp. 85-111.

SCHERR, F.; SUGRUE, T. y WARD, J. (1993) Financing the small firm start-up: determinants of debt use. The Journal of Small Business Finance, vol. 3, № 1, pp. 17-36.

SERVER, R. y CAPÓ, J. (2009) La responsabilidad social empresarial en un contexto de crisis. Repercusión en las sociedades cooperativas. Ciriec - Revista de Economía Pública, Social y Cooperativa, vol. 65, pp. 7-31.

SHRADER, C.; BLACKBURN, V. y ILES, P. (1997) Women in management and firm financial value: An exploratory study. Journal of Managerial Issues, vol. 9, pp. 355-372.

SMITH, N.; SMITH, V. y VERNER, N. (2006) Do women in top management affect firm performance? A panel study of 2,500 Danish firms. International Journal of Productivity and Performance Management, vol. 55, № 7, pp. 569-593.

SPEAR, R. (2004) Governance in democratic member-based organizations, Annals of public and cooperative economics, vol. 75, № 1, pp. 33-59.

TERJESEN, S.; SEALY, R. y SINGH, V. (2009) Women directors on corporate boards: A review and research agenda. Corporate Governance: An International Review, vol. 17, $\mathrm{N}^{\mathrm{o}}$ 3, pp. 320-337.

VERHEUL, I. y THURIK, R. (2001) Start-up capital: Does gender matter? Small Business Economics, vol. 16, № 4, pp. 329-346.

VOSSEN, R. (1998) Relative strengths and weaknesses of small firms in innovation. International Small Business Journal, vol. 16, No 3, pp. 88-94.

WADDOCK, S. y GRAVES, S. (1997) The corporate social performance. Strategic Management Journal, vol. 8, № 4, pp. 303-319.

WELBOURNE, T.; CYCYOTA, C. y FERRANTE, C. (2007) Wall Street reaction to women in IPOs: An examination of gender diversity in top management teams. Group and Organization Management, vol. 32, № 5, pp. 524-47. 
ZELECHOWSKI, D. y BILIMORIA, D. (2004) Characteristics of women and men corporate inside directors. Corporate Governance: An International Review, vol. 12, No 3, pp. 337342. 